\title{
Nouveau guide pour les professionnels de la santé
}

\author{
Valérie Clerc \\ Secrétaire générale de I'ASSM
}

Le questionnement sur l'avenir du système de santé et les défis posés par la médecine personnalisée font partie des thèmes en vogue dans les milieux médiatiques, académiques et politiques. En tant qu'actrice du domaine, l'Académie Suisse des Sciences Médicales (ASSM) n'échappe pas à cette tendance. Convaincue que les professionnels de la santé devraient disposer de connaissances de base communes en matière de médecine personnalisée, elle publie un document intitulé «Médecine personnalisée. Bases pour la formation interprofessionnelle prégraduée, postgraduée et continue des professionnels de la santé».

Il y a plus d'un siècle, le médecin anglais Archibald E. Garrod a reconnu l'importance de l'«individualité chimique» de l'être humain. Toutefois, il a fallu attendre plusieurs décennies pour pouvoir appliquer ce constat au service de la santé des patients. Grâce aux progrès des sciences biomédicales et des technologies de l'information, nous disposons aujourd'hui d'une quantité énorme de données exploitées pour identifier au plus tôt les risques de maladie, adapter les traitements médicaux aux particularités de groupes de patients spécifiques et développer des stratégies de santé pour la population.

Le terme «médecine personnalisée» n'est pas des plus heureux. La médecine a, de tout temps, été personnalisée. Depuis toujours, les professionnels de la santé adaptent leurs traitements aux patients. Dans la préface qu'il rédige en tant que président de l'ASSM, Daniel

\section{En savoir plus}

La nouvelle publication de I'ASSM s'inscrit dans une série de projets en lien avec la médecine personnalisée que I'ASSM conduit seule ou en collaboration avec d'autres membres des Académies suisses des sciences. Sous l'égide de I'ASSM, le Swiss Personalized Health Network (SPHN) a reçu pour mission de la Confédération de rendre les données de santé exploitables pour la recherche en Suisse et d'encourager le développement de la médecine et de la santé personnalisées. Dans le même temps, I'ASSM considère qu'une information neutre concernant les chances et les risques de la médecine personnalisée doit être apportée à la population. Le portail thématique "Santé personnalisée», géré par le Forum Recherche génétique de l'Académie des sciences naturelles (SCNAT), et le projet de dialogue "L'humain sur mesure» de la fondation Science et Cité sont des exemples d'initiatives soutenues par I'ASSM et qui poursuivent cet objectif.
Scheidegger va au-delà des querelles terminologiques et explique ce qui a changé: «L’expérience du spécialiste individuel qui ne connaît qu'un nombre limité de patientes et de patients ne suffit plus. Grâce aux énormes banques de données, des sous-groupes diagnostiques permettant de tester un traitement prometteur peuvent être constitués. Les résultats concernant les succès cliniques à long terme et les effets secondaires deviennent ainsi plus rapidement accessibles à tous.»

Pour une mise en œuvre réussie, la médecine personnalisée demande l'acquisition de nouvelles compétences par les professionnels de la santé. Ainsi, le double profil de médecin et bio-informaticien est très recherché. Comme l'explique le Prof. Antoine Geissbuhler dans le journal de l'Université de Genève Campus, l'enseignement de la médecine doit s'adapter à ces changements. Il doit désormais apprendre aux étudiants à utiliser des programmes informatiques qui trieront et analyseront de grandes quantités de données, à comprendre leur fonctionnement, mais aussi leurs limites. Ainsi, Genève prépare un cours en ligne (MOOC) consacré à ce sujet, conçu pour les étudiants de $3^{\mathrm{e}}$ année en biologie et médecine [1].

\section{Approche interprofessionnelle}

Le développement d'une médecine «sur mesure», axée sur la personnalisation, la prévision, la prédiction et la participation, aura un impact non seulement sur la formation des médecins, mais sur celle de tous les professionnels de la santé. C'est dans cet état d'esprit que 
l'ASSM a conçu sa publication pour la formation des professionnels de la santé en médecine personnalisée. Sous la présidence de la Prof. Anita Rauch, cheffe de l'Institut de médecine génétique à l'Université de Zurich et membre du Comité de direction de l'ASSM, se

\section{La médecine personnalisée constitue un terrain idéal pour penser la formation d'emblée dans une perspective interprofessionnelle.}

sont réunis des délégués des associations professionnelles de pharmaciens et de physiothérapeutes, des membres de sociétés médicales, des professeurs HES du domaine des soins infirmiers, des généticiens, des médecins de famille, sans oublier les représentants de patients.

La médecine personnalisée constitue un terrain idéal pour penser la formation d'emblée dans une perspective interprofessionnelle. Une telle approche correspond d'ailleurs au modèle préconisé par l'ASSM dans sa publication de 2007 sur les futurs profils professionnels des médecins et des infirmiers, qui prévoit, au-delà des éléments propres au profil professionnel, des éléments de base à acquérir en commun [2]. Elle aura entre autres l'avantage que les différents professionnels de la santé "parlent le même langage» une fois qu'ils se retrouvent dans un colloque ou au lit du patient et que leurs compétences s'additionneront pour mettre en œuvre le traitement le plus approprié possible.

\section{Reflet de l'état actuel des connaissances}

La présente publication a été pensée comme un document de référence présentant l'état actuel des connaissances pour la formation prégraduée, postgraduée et continue en médecine personnalisée. Le champ couvert est très large. Après une introduction sur les définitions et les concepts, qui met en évidence aussi bien les possibilités que les limites, sa signification pour les associations de patients et l'économie de la santé, le document s'intéresse aux aspects médicaux: génétique, oncologie, pharmacogénomique, sciences des don- nées, santé publique. Le dernier chapitre est consacré aux aspects légaux, éthiques et psychosociaux. Un vaste glossaire permet à un public non averti de se familiariser avec le jargon propre au domaine.

Tout en étant consciente que la durée de vie d'une telle publication est limitée, l'ASSM espère qu'elle contribuera à diffuser les contenus en matière de médecine personnalisée dans les hautes écoles formant les futurs professionnels de santé, que ces dernières se les approprient et les fassent évoluer. Il en va de même pour la formation postgrade et continue. Comme l'écrit Daniel Scheidegger, «ces prochaines années, la médecine personnalisée gagnera de plus en plus en importance. L'avenir nous dira si les grands espoirs se réaliseront. Il sera intéressant de consulter ce document dans une dizaine d'années et de voir quelles prévisions qu'elles soient positives ou négatives - se sont réalisées.» La nouvelle publication peut être téléchargée en version intégrale ou chapitre par chapitre sur le site web de l'ASSM: assm.ch/bases-medecine-personnalisee

\section{Références}

1 Campus. Le magazine scientifique de l'Université de Genève, $\mathrm{n}^{\circ} 138$, septembre 2019, pp. 19-20.

2 ASSM. Les futurs profils professionnels des médecins et des infirmiers. Rapport et commentaire. 2007 et 2011, pp. 10-11.

\section{L'essentiel en bref}

- L'ASSM est convaincue de l'importance pour tous les professionnels de la santé de disposer de connaissances de base communes en matière de médecine personnalisée; c'est pourquoi elle publie un document qui se veut utile pour la formation interprofessionnelle prégraduée, postgraduée et continue.

- Cette publication a été pensée comme un document de référence présentant l'état actuel des connaissances.

- Le document s'intéresse aux aspects médicaux tels que la génétique, l'oncologie, la pharmacogénomique, les sciences des données et la santé publique.

- En complément à la partie médicale, le document contient une introduction sur les définitions et les concepts, un chapitre sur les aspects juridiques, éthiques et psychosociaux ainsi qu'un glossaire complet. 